\title{
Time-Varying Input and State Delay Compensation for Uncertain Nonlinear Systems
}

\author{
Rushikesh Kamalapurkar, Nicholas Fischer, Serhat Obuz, and Warren E. Dixon
}

\begin{abstract}
A robust controller is developed for uncertain, second-order nonlinear systems subject to simultaneous unknown, time-varying state delays and known, time-varying input delays in addition to additive, sufficiently smooth disturbances. An integral term composed of previous control values facilitates a delay-free open-loop error system and the development of the feedback control structure. A stability analysis based on Lyapunov-Krasovskii (LK) functionals guarantees uniformly ultimately bounded tracking under the assumption that the delays are bounded and slowly varying.
\end{abstract}

\section{INTRODUCTION}

Numerous control techniques exist for linear systems with constant input delays (cf. [1]-[3] and references therein). Many of these results are extensions of classic Smith predictors [4], Artstein model reduction [5], or finite spectrum assignment [6]. Results that focus on simultaneous constant state and input delays for linear systems are provided in [7][9]. Extensions of linear control techniques to time-varying input delays are also available [10]-[15].

For nonlinear systems, controllers considering constant [16]-[23] and time-varying [20], [24]-[33] state delays have been recently developed. However, linear results considering delayed inputs are far less prevalent, especially for systems with model uncertainties and/or disturbances. Examples of these include constant input delay results in [34]-[46] and time-varying input delay results based on LMI conditions [47], [48], backstepping [49]-[51] and other robust techniques [52]. Even more unique are results that consider both state and input delays in nonlinear systems. Recently in [51], the predictorbased techniques in [7] were extended to nonlinear systems with time-varying delays in the state and/or the input utilizing a backstepping transformation to construct a predictor-based compensator. The development in [51] requires knowledge of the plant dynamics and assumes that the plant is disturbancefree.

In this paper, we expand our previous time-varying input delay result [52] in two directions: a) Utilizing techniques for constant input-delayed systems first introduced in [46], we consider time-varying input delays in a nonlinear plant,

Rushikesh Kamalapurkar, Nicholas Fischer, Serhat Obuz, and Warren E. Dixon are with the Department of Mechanical and Aerospace Engineering, University of Florida, Gainesville, FL, USA. Email: rkamalapurkar@ufl.edu, nic.r.fischer@gmail.com, \{serhat.obuz,wdixon\}@ufl.edu.

This research is supported in part by NSF award numbers 1161260 and 1217908, ONR grant number N00014-13-1-0151, and a contract with the AFRL Mathematical Modeling and Optimization Institute. Any opinions, findings and conclusions or recommendations expressed in this material are those of the authors and do not necessarily reflect the views of the sponsoring agency. and b) we add the ability to compensate for simultaneous arbitrarily large unknown time-varying state delays based on the techniques in [33]. Robust control methods are developed to compensate for the unknown time-varying state delays. To compensate for the input delay, an integral term composed of previous control values is used to yield a delay-free openloop system. A Lyapunov-based stability analysis motivated by Lyapunov-Krasovskii (LK) functionals demonstrates the ability to achieve uniformly ultimately bounded tracking in the presence of model uncertainty, additive sufficiently smooth disturbances, and simultaneous time-varying state and input delays. The result is based on the assumption that the unknown state delay is bounded and slowly varying. Improving on the result in [52], we relax previous sufficient conditions on the control gains that required knowledge of the second derivative of the input delay.

\section{DYNAMIC SYSTEM}

Consider a class of second-order (Euler-Lagrange-like) nonlinear systems given by:12

$$
\ddot{x}=f(x, \dot{x}, t)+g\left(x\left(t-\tau_{s}\right), \dot{x}\left(t-\tau_{s}\right), t\right)+d+u_{\tau_{i}},
$$

where $x, \dot{x} \in \mathbb{R}^{n}$ are the system states, $u \in \mathbb{R}^{n}$ is the control input, $f: \mathbb{R}^{n} \times \mathbb{R}^{n} \times[0, \infty) \rightarrow \mathbb{R}^{n}$ is an unknown function, uniformly bounded in $t, g: \mathbb{R}^{n} \times \mathbb{R}^{n} \times[0, \infty) \rightarrow \mathbb{R}^{n}$ is an unknown function with delayed internal state, uniformly bounded in $t, d \in \mathbb{R}^{n}$ denotes a sufficiently smooth disturbance (e.g., unmodeled effects), and $\tau_{i}, \tau_{s} \in[0, \infty)$ denote time-varying, non-negative input and state delays, respectively.

The subsequent development is based on the assumption that $x$ and $\dot{x}$ are measurable outputs. Throughout the paper, a time-dependent delayed function is denoted as

$$
\zeta_{\tau} \triangleq \begin{cases}\zeta(t-\tau) & t-\tau>t_{0} \\ 0 & t-\tau \leq t_{0}\end{cases}
$$

where $t_{0}$ denotes the initial time. Thus, $u_{\tau_{i}}$ is defined by

$$
u_{\tau_{i}} \triangleq \begin{cases}u\left(t-\tau_{i}\right) & t-\tau_{i}>t_{0} \\ 0 & t-\tau_{i} \leq t_{0} .\end{cases}
$$

\footnotetext{
${ }^{1}$ The result in this paper can be extended to $n^{\text {th }}$-order nonlinear systems following a similar development to that presented in [32].

${ }^{2}$ For notational brevity, unless otherwise specified, the domain of all the functions is assumed to be $\mathbb{R}_{\geq 0}$. Furthermore, unless otherwise specified, all mathematical quantities are assumed to be time-varying and time-dependence is suppressed in equations and definitions. For example, the trajectory $x$ : $\mathbb{R}_{>0} \rightarrow \mathbb{R}^{n}$ is defined by abuse of notation as $x \in \mathbb{R}^{n}$ and unless otherwise specified, an equation (inequality) of the form $f+h(y, t)=(\leq) g(x)$ is interpreted as $f(t)+h(y(t), t)=(\leq) g(x(t))$ for all $t \in \mathbb{R}_{\geq 0}$.
} 
Additionally, $\|\cdot\|$ denotes the Euclidean norm of a vector and the following assumptions will be exploited.

Assumption 1. Each of the functions $f$ and $g$, along with their first and second partial derivatives, is bounded on each subset of its domain of the form $K \times[0, \infty)$, where $K \subset$ $\mathbb{R}^{n} \times \mathbb{R}^{n}$ is compact. Furthermore, given such compact $K$, the corresponding bound is known.

Assumption 2. The nonlinear disturbance term and its time derivative are bounded by known constants 3

Assumption 3. The desired trajectory $x_{d} \in \mathbb{R}^{n}$ is designed such that $x_{d}^{(i)} \in \mathbb{R}^{n}, \forall i=0,1, \ldots, 3$ exist and are bounded by known positive constants, where the superscript $(i)$ denotes the $i^{\text {th }}$ time derivative 4

Assumption 4. The input and state delays are bounded such that $0 \leq \tau_{i} \leq \varphi_{i_{1}}$ and $0 \leq \tau_{s} \leq \varphi_{s_{1}}$, and the rate of change of the delays are bounded such that $\left|\dot{\tau}_{i}\right| \leq \varphi_{i_{2}}<1$ and $\left|\dot{\tau}_{s}\right| \leq \varphi_{s_{2}}<1$ where $\varphi_{j} \in \mathbb{R}^{+} \forall j=i_{1}, i_{2}, s_{1}, s_{2}$ are known constants. Furthermore, the bounds on the input delay satisfy $\varphi_{i_{1}}+\varphi_{i_{2}}<1$. The state delay is assumed to be unknown, while the input delay is assumed to be known.

Remark 1. In Assumption 4 the slowly time-varying constraint (i.e., $\left|\dot{\tau}_{i, s}\right| \leq \varphi_{i_{2}, s_{2}}<1$ ) is common to results which utilize classical LK functionals to compensate for timevarying time-delays [14]. Knowledge of the state delays in the system is not required; however, the input delays present a more significant challenge. Although the controller requires the input delay to be known so that the interval of past control values can be properly sized, numerical simulations illustrate robustness to uncertainties in the input delay.

\section{Control Objective}

The objective is to design a controller that will ensure the system state $x$ of the system in (1) tracks a desired state trajectory. To quantify the control objective, a tracking error, denoted by $e_{1} \in \mathbb{R}^{n}$, is defined as

$$
e_{1} \triangleq x_{d}-x
$$

To facilitate the subsequent analysis, two auxiliary tracking errors $e_{2}, r \in \mathbb{R}^{n}$ are defined as [46]

$$
\begin{aligned}
& e_{2} \triangleq \dot{e}_{1}+\alpha_{1} e_{1}, \\
& r \triangleq \dot{e}_{2}+\alpha_{2} e_{2}+e_{u},
\end{aligned}
$$

where $\alpha_{1}, \alpha_{2} \in \mathbb{R}$ denote constant positive control gains, and $e_{u} \in \mathbb{R}^{n}$ denotes the mismatch between the delayed control input and the computed control input, defined as 5

$$
e_{u} \triangleq u_{\tau_{i}}-u
$$

${ }^{3}$ Many practical disturbance terms are continuous including friction (see [53], [54]), wind disturbances, wave/ocean disturbances, etc.

${ }^{4}$ Many guidance and navigation applications utilize smooth, high-order differentiable desired trajectories. Curve fitting methods can also be used to generate sufficiently smooth time-varying trajectories.

${ }^{5}$ Let $h \triangleq \max \left(t_{0}, t-\tau_{i}\right)$. Then, $h:[0, \infty) \rightarrow[0, \infty)$ is continuous. Further, since $u\left(t_{0}\right)=0, u_{\tau_{i}}=u(h)$, and $e_{u}=u(h)-u$. Hence, $e_{u}$ is a continuous function of time if $u$ is a continuous function of time, and $e_{u}\left(t_{0}\right)=0$.
The auxiliary signal $e_{u}$ injects a delay-free control input into the error system development. In contrast to the development in [52], the term in (5]) is embedded in a higher order derivative (i.e., $r$ instead of $e_{2}$ ). Functionally, $e_{u}$ still injects an integral of past control values into the open-loop system; however, the development introduces fewer cross-terms. The auxiliary signal $r$ is introduced to facilitate the stability analysis and is not used in the control design since the expression in (4) depends on the unmeasurable state $\ddot{x}$. The structure of the error systems is motivated by the need to inject and cancel terms in the subsequent stability analysis as demonstrated in Section IV

Using (11), 2), (3), and (5) to eliminate the delayed input term, (4) can be represented as

$$
r=S_{1}+S_{2}-u,
$$

where the auxiliary functions $S_{1} \in \mathbb{R}^{n}$ and $S_{2} \in \mathbb{R}^{n}$ are defined as

$$
\begin{aligned}
S_{1} & \triangleq f\left(x_{d}, \dot{x}_{d}, t\right)-f(x, \dot{x}, t)+g\left(x_{d \tau_{s}}, \dot{x}_{d \tau_{s}}, t\right) \\
& -g\left(x_{\tau_{s}}, \dot{x}_{\tau_{s}}, t\right)+\alpha_{1} \dot{e}_{1}+\alpha_{2} e_{2}, \\
S_{2} & \triangleq \ddot{x}_{d}-f\left(x_{d}, \dot{x}_{d}, t\right)-g\left(x_{d \tau_{s}}, \dot{x}_{d \tau_{s}}, t\right)-d .
\end{aligned}
$$

Based on (6) and the subsequent stability analysis,

$$
u \triangleq\left(k_{s}+1\right)\left(e_{2}-e_{2}\left(t_{0}\right)\right)+v
$$

where $v \in \mathbb{R}^{n}$ is the solution to the following differential equation

$$
\dot{v}=\left(k_{s}+1\right)\left(\alpha_{2} e_{2}+e_{u}\right), v\left(t_{0}\right)=0,
$$

and $k_{s} \in \mathbb{R}$ is a positive constant control gain.

The closed-loop error system can be developed by taking the time derivative of (6) and substituting for (4) and the time derivative of (7) to yield

$$
\dot{r}=\tilde{N}+N_{d}-e_{2}-\left(k_{s}+1\right) r,
$$

where $\tilde{N} \in \mathbb{R}^{n}$ and $N_{d} \in \mathbb{R}^{n}$ are defined as

$$
\begin{aligned}
& \tilde{N} \triangleq \dot{S}_{1}+e_{2}, \\
& N_{d} \triangleq \dot{S}_{2} .
\end{aligned}
$$

The control design in (7) and (8) is motivated by the desire to eliminate the delayed input, yielding the closed-loop error system in (9). The structure of 90 is advantageous because it facilitates the stability analysis by segregating terms that can be upper bounded by a state-dependent term and terms that can be upper bounded by constants. Based on Assumptions 2 and 3 , the following inequalities can be developed from the expression in (11):

$$
\left\|N_{d}\right\| \leq \zeta_{N_{d 1}}
$$

where $\zeta_{N_{d 1}} \in \mathbb{R}$, is a known positive constant. The Mean Value Theorem can be utilized to find an upper bound for the expression in (10) as [55, Appendix A]

$$
\|\tilde{N}\| \leq \rho_{1}(\|z\|)\|z\|+\rho_{2}\left(\left\|z_{\tau_{s}}\right\|\right)\left\|z_{\tau_{s}}\right\|,
$$

where $z \in \mathbb{R}^{4 n}$ denotes the vector

$$
z \triangleq\left[e_{1}^{T}, e_{2}^{T}, r^{T}, e_{u}^{T}\right]^{T},
$$


and the bounding terms $\rho_{1}, \rho_{2}:[0, \infty) \rightarrow[0, \infty)$ are positive, non-decreasing and radially unbounded functions 6 The upper bound for the auxiliary function $\tilde{N}$ is segregated into delayfree and delay-dependent bounding functions to eliminate the delayed terms with the use of an LK term in the stability analysis.

To facilitate the subsequent stability analysis, several auxiliary terms are introduced. Let $\rho:[0, \infty) \rightarrow[0, \infty)$ be an auxiliary bounding function defined as

$$
\rho(\|z\|)=\sqrt{\left(\gamma_{1}+2 \gamma_{2} \varphi_{s_{1}}\right) \rho_{2}^{2}(\|z\|)+3 \rho_{1}^{2}(\|z\|)},
$$

where $\gamma_{1}$ and $\gamma_{2}$ are positive adjustable constants, and let $z \in$ $\mathbb{R}^{3 n}$ be defined as

$$
\dot{z} \triangleq\left[e_{1}^{T}, e_{2}^{T}, r^{T}\right]^{T} .
$$

Auxiliary bounding constants $\sigma, \delta \in \mathbb{R}$ are defined as

$$
\begin{aligned}
& \sigma \triangleq \frac{1}{2} \min \left\{\frac{\alpha_{1}}{2}, \frac{\alpha_{2}}{2}, 1, \frac{\omega\left(1-\varphi_{i_{2}}\right)}{6 \varphi_{i_{1}}}\right\}, \\
& \delta \triangleq \frac{1}{2} \min \left\{\sigma, \frac{\omega\left(1-\varphi_{i_{2}}\right)}{3 \varphi_{i_{1}}}, \frac{\left(1-\varphi_{i_{2}}\right)}{3 \varphi_{i_{1}}},\right. \\
& \left.\frac{\gamma_{2}\left(1-\varphi_{s_{2}}\right)}{\gamma_{1}}, \frac{\left(1-\varphi_{s_{2}}\right)}{2 \varphi_{s_{1}}}\right\},
\end{aligned}
$$

where $\omega \in \mathbb{R}$ is a known, positive, adjustable constant.

Let

$$
\mathscr{D} \triangleq\left\{\xi \in \mathbb{R}^{3 n+4} \mid\|\xi\|<\inf \left\{\rho^{-1}\left(\left[\sqrt{2 k_{s} \sigma}, \infty\right)\right)\right\}\right\},
$$

and

$$
\mathcal{S}_{\mathscr{D}} \triangleq\left\{\xi \in \mathscr{D} \mid\|\xi\|<\sqrt{\frac{1}{2}} \inf \left\{\rho^{-1}\left(\left[\sqrt{2 k_{s} \sigma}, \infty\right)\right)\right\}\right\},
$$

where, for a set $A \subset \mathbb{R}$, the inverse image $\rho^{-1}(A) \subset \mathbb{R}$ is defined as $\rho^{-1}(A) \triangleq\{a \in \mathbb{R} \mid \rho(a) \in A\}$. Furthermore, let the functions $P_{L K}:[0, \infty) \rightarrow[0, \infty), Q_{L K}:[0, \infty) \rightarrow$ $[0, \infty), R_{L K}:[0, \infty) \rightarrow[0, \infty)$, and $S_{L K}:[0, \infty) \rightarrow[0, \infty)$ be defined as

$$
\begin{aligned}
& P_{L K} \triangleq \varphi_{i_{1}} \int_{t-\tau_{i}}^{t}\|\dot{u}(\theta)\|^{2} d \theta, \\
& Q_{L K} \triangleq \omega \int_{t-\tau_{i}}^{t}\left(\int_{s}^{t}\|\dot{u}(\theta)\|^{2} d \theta\right) d s, \\
& R_{L K} \triangleq \frac{\gamma_{1}}{2 k_{s}} \int_{t-\tau_{s}}^{t} \rho_{2}^{2}(\|z(\sigma)\|)\|z(\sigma)\|^{2} d \sigma, \\
& S_{L K} \triangleq \frac{\gamma_{2}}{k_{s}} \int_{t-\tau_{s}}^{t}\left(\int_{s}^{t} \rho_{2}^{2}(\|z(\sigma)\|)\|z(\sigma)\|^{2} d \sigma\right) d s .
\end{aligned}
$$

Additionally, let $y \in \mathbb{R}^{3 n+4}$ be defined as

$$
y \triangleq\left[\begin{array}{lllll}
\dot{z}^{T} & \sqrt{P_{L K}} & \sqrt{Q_{L K}} & \sqrt{R_{L K}} & \sqrt{S_{L K}}
\end{array}\right]^{T} .
$$

${ }^{6}$ For some classes of systems, the bounding functions $\rho_{1}$ and $\rho_{2}$ could be selected as constants. For these classes of systems, a global uniformly ultimately bounded result can be obtained as described in Remark 2

${ }^{7}$ The construction of $P_{L K}, Q_{L K}, R_{L K}$, and $S_{L K}$ is based on $\mathrm{LK}$ functionals. However, in this result, they are to be interpreted as time-varying signals that are a part of the system state.

\section{Stability ANALysis}

Theorem 1. Given the dynamics in (17), provided the control gains are selected based on the following sufficient conditions

$$
\alpha_{1}>1, \alpha_{2}>2, \gamma_{1}>\frac{1}{\left(1-\varphi_{s_{2}}\right)}, \omega>\frac{3 \varphi_{i_{1}}}{\left(1-\varphi_{i_{2}}\right)},
$$

and the input delay is small enough so that there exists a gain $k_{s}$ that satisfies 8

$$
\begin{aligned}
\varphi_{i_{1}} & <\frac{k_{s}}{6(\omega+1)\left(k_{s}+1\right)^{2}}, \\
\frac{3 \zeta_{N_{d 1}}^{2}}{k_{s} \delta} & <\left(\inf \left\{\rho^{-1}\left(\left[\sqrt{2 k_{s} \sigma}, \infty\right)\right)\right\}\right)^{2},
\end{aligned}
$$

the controller given in (7) and (8) ensures uniformly ultimately bounded tracking in the sense that $\lim \sup _{t \rightarrow \infty}\|y\| \leq$ $\sqrt{\frac{3 \zeta_{N_{d 1}}^{2}}{k_{s} \delta}}$, provided $y\left(t_{0}\right) \in \mathcal{S}_{\mathscr{D}}$.

Proof: Let $V: \mathscr{D} \rightarrow \mathbb{R}$ be a candidate Lyapunov function defined as

$$
\begin{aligned}
V \triangleq \frac{1}{2} e_{1}^{T} e_{1}+\frac{1}{2} e_{2}^{T} e_{2}+\frac{1}{2} r^{T} r+P_{L K} & +Q_{L K} \\
& +R_{L K}+S_{L K},
\end{aligned}
$$

which satisfies the following inequalities:

$$
\frac{1}{2}\|y\|^{2} \leq V(y) \leq\|y\|^{2} .
$$

The time derivative of 27) can be found by applying the Leibniz Rule to (19), (20), (21) and 222), and by substituting (2)-(4), (7), and (9), yielding

$$
\begin{aligned}
\dot{V} & =e_{1}^{T}\left(e_{2}-\alpha_{1} e_{1}\right)+e_{2}^{T}\left(r-\alpha_{2} e_{2}-e_{u}\right) \\
& +r^{T}\left(\tilde{N}+N_{d}-e_{2}-\left(k_{s}+1\right) r\right) \\
& +\left(\omega \tau_{i}+\varphi_{i_{1}}\right)\left(k_{s}+1\right)^{2}\|r\|^{2}-\varphi_{i_{1}}\left(1-\dot{\tau}_{i}\right)\left\|\dot{u}_{\tau_{i}}\right\|^{2} \\
& -\omega\left(1-\dot{\tau}_{i}\right) \int_{t-\tau_{i}}^{t}\|\dot{u}(\theta)\|^{2} d \theta \\
& +\left(\frac{\gamma_{1}}{2 k_{s}}+\frac{\gamma_{2}}{k_{s}} \tau_{s}\right) \rho_{2}^{2}(\|z\|)\|z\|^{2} \\
& -\frac{\gamma_{1}\left(1-\dot{\tau}_{s}\right)}{2 k_{s}} \rho_{2}^{2}\left(\left\|z_{\tau_{s}}\right\|\right)\left\|z_{\tau_{s}}\right\|^{2} \\
& -\frac{\gamma_{2}}{k_{s}}\left(1-\dot{\tau}_{s}\right) \int_{t-\tau_{s}}^{t} \rho_{2}^{2}(\|z(\theta)\|)\|z(\theta)\|^{2} d \theta
\end{aligned}
$$

Using (4), (12), (13), the inequality $\dot{\tau}_{s}<1$ and Young's Inequality to show that $\left\|e_{1}^{T} e_{2}\right\| \leq \frac{1}{2}\left\|e_{1}\right\|^{2}+\frac{1}{2}\left\|e_{2}\right\|^{2}$, $\left\|e_{2}^{T} e_{u}\right\| \leq \frac{1}{2}\left\|e_{2}\right\|^{2}+\frac{1}{2}\left\|e_{u}\right\|^{2}$ and $\|r\| \rho_{2}\left(\left\|z_{\tau_{s}}\right\|\right)\left\|z_{\tau_{s}}\right\| \leq$ $\frac{k_{s}}{2}\|r\|^{2}+\frac{1}{2 k_{s}} \rho_{2}^{2}\left(\left\|z_{\tau_{s}}\right\|\right)\left\|z_{\tau_{s}}\right\|^{2}$, the expression in (29) can be upper bounded as

$$
\begin{aligned}
\dot{V} & \leq-\alpha_{1}\left\|e_{1}\right\|^{2}-\alpha_{2}\left\|e_{2}\right\|^{2}-\left(\frac{k_{s}}{2}+1\right)\|r\|^{2} \\
& +\frac{1}{2}\left\|e_{1}\right\|^{2}+\left\|e_{2}\right\|^{2}+\frac{1}{2}\left\|e_{u}\right\|^{2}+\|r\| \rho_{1}(\|z\|)\|z\|
\end{aligned}
$$

${ }^{8}$ Since $\delta$ increases with increasing $k_{s}$, the left-hand side of 26 decreases with increasing $k_{s}$. Since $\rho$ is a nondecreasing function, the right-hand side of 26 is nondecreasing with respect to $k_{s}$. Hence, 26 can be satisfied for some $k_{s}$. Furthermore, for any given $k_{s}, 25$ is satisfied if the delay is small enough. 


$$
\begin{aligned}
& +\frac{1}{2 k_{s}} \rho_{2}^{2}\left(\left\|z_{\tau_{s}}\right\|\right)\left\|z_{\tau_{s}}\right\|^{2}-\frac{\gamma_{1}\left(1-\dot{\tau}_{s}\right)}{2 k_{s}} \rho_{2}^{2}\left(\left\|z_{\tau_{s}}\right\|\right)\left\|z_{\tau_{s}}\right\|^{2} \\
& +\varphi_{i_{1}}(1+\omega)\left(k_{s}+1\right)^{2}\|r\|^{2}+\|r\| \zeta_{N_{d 1}} \\
& -\omega\left(1-\dot{\tau}_{i}\right) \int_{t-\tau_{i}}^{t}\|\dot{u}(\theta)\|^{2} d \theta \\
& +\left(\frac{\gamma_{1}}{2 k_{s}}+\frac{\gamma_{2}}{k_{s}} \tau_{s}\right) \rho_{2}^{2}(\|z\|)\|z\|^{2} \\
& -\frac{\gamma_{2}}{k_{s}}\left(1-\dot{\tau}_{s}\right) \int_{t-\tau_{s}}^{t} \rho_{2}^{2}(\|z(\theta)\|)\|z(\theta)\|^{2} d \theta
\end{aligned}
$$

Completing the squares for $r$, utilizing the inequalities

$$
\begin{gathered}
\left\|e_{u}\right\|^{2} \leq \tau_{i} \int_{t-\tau_{i}}^{t}\|\dot{u}(\theta)\|^{2} d \theta \\
-\int_{t-\tau_{i}}^{t}\|\dot{u}(\theta)\|^{2} d \theta \leq \\
-\frac{1}{\tau_{i}} \int_{t-\tau_{i}}^{t}\left(\int_{s}^{t}\|\dot{u}(\theta)\|^{2} d \theta\right) d s=-\frac{Q_{L K}}{\omega \tau_{i}}, \\
-\int_{t-\tau_{s}}^{t} \rho_{2}^{2}(\|z(\theta)\|)\|z(\theta)\|^{2} d \theta \leq \\
-\frac{1}{\tau_{s}} \int_{t-\tau_{s}}^{t}\left(\int_{s}^{t} \rho_{2}^{2}(\|z(\theta)\|)\|z(\theta)\|^{2} d \theta\right) d s= \\
-\frac{k_{s} S_{L K}}{\gamma_{2} \tau_{s}},
\end{gathered}
$$

and 26, 19) and 21, 30, can be rewritten as

$$
\begin{aligned}
\dot{V} & \leq-\frac{\alpha_{1}}{2}\left\|e_{1}\right\|^{2}-\frac{\alpha_{2}}{2}\left\|e_{2}\right\|^{2}-\|r\|^{2}-\frac{\omega\left(1-\dot{\tau}_{i}\right)}{6 \tau_{i}}\left\|e_{u}\right\|^{2} \\
& -\left(\frac{\alpha_{1}}{2}-\frac{1}{2}\right)\left\|e_{1}\right\|^{2}-\left(\frac{\alpha_{2}}{2}-1\right)\left\|e_{2}\right\|^{2} \\
& -\left(\frac{k_{s}}{6}-\varphi_{i_{1}}(1+\omega)\left(k_{s}+1\right)^{2}\right)\|r\|^{2} \\
& -\left(\frac{\omega\left(1-\dot{\tau}_{i}\right)}{6 \tau_{i}}-\frac{1}{2}\right)\left\|e_{u}\right\|^{2} \\
& -\left(\frac{\gamma_{1}\left(1-\dot{\tau}_{s}\right)}{2 k_{s}}-\frac{1}{2 k_{s}}\right) \rho_{2}^{2}\left(\left\|z_{\tau_{s}}\right\|\right)\left\|z_{\tau_{s}}\right\|^{2} \\
& +\frac{1}{2 k_{s}}\left(3 \rho_{1}^{2}(\|z\|)+\left(\gamma_{1}+2 \gamma_{2} \varphi_{s_{1}}\right) \rho_{2}^{2}(\|z\|)\right)\|z\|^{2} \\
& -\frac{\omega\left(1-\dot{\tau}_{i}\right)}{3 \varphi_{i_{1}}} P_{L K}-\frac{\left(1-\dot{\tau}_{i}\right)}{3 \tau_{i}} Q_{L K}-\frac{\gamma_{2}\left(1-\dot{\tau}_{s}\right)}{\gamma_{1}} R_{L K} \\
& -\frac{\left(1-\dot{\tau}_{s}\right)}{2 \tau_{s}} S_{L K}+\frac{3 \zeta_{N_{d 1}}^{2}}{2 k_{s}} .
\end{aligned}
$$

If the conditions in (24) are satisfied, based on the inequalities $\|z\|^{2} \geq\|z\|^{2}$ and $\|z\| \leq\|y\|$, the expression in (31) reduces to

$$
\begin{aligned}
\dot{V} & \leq-\sigma\|\dot{z}\|^{2}-\frac{\omega\left(1-\varphi_{i_{2}}\right)}{3 \varphi_{i_{1}}} P_{L K}-\frac{\left(1-\varphi_{i_{2}}\right)}{3 \varphi_{i_{1}}} Q_{L K} \\
& -\frac{\gamma_{2}\left(1-\varphi_{s_{2}}\right)}{\gamma_{1}} R_{L K}-\frac{\left(1-\varphi_{s_{2}}\right)}{2 \varphi_{s_{1}}} S_{L K}+\frac{3 \zeta_{N_{d 1}}^{2}}{2 k_{s}} \\
& \leq-\delta\|y\|^{2}, \forall\|y\| \geq \sqrt{\frac{3 \zeta_{N_{d 1}}^{2}}{2 k_{s} \delta}}
\end{aligned}
$$

provided $y \in \mathscr{D}$, where $\rho(\|z\|), \sigma$, and $\delta$ were introduced in (15), (17) and (18). Using (26), (28), and (32), Theorem 4.18 in [56] can be invoked to conclude that $y$ is uniformly ultimately bounded in the sense that $\limsup _{t \rightarrow \infty}\|y\| \leq \sqrt{\frac{3 \zeta_{N_{d 1}}^{2}}{k_{s} \delta}}$, provided $y\left(t_{0}\right) \in \mathcal{S}_{\mathscr{D}}$.

Since $e_{1}, e_{2}, r \in \mathcal{L}_{\infty}$, from (6), $u \in \mathcal{L}_{\infty}$, which implies $u_{\tau i} \in \mathcal{L}_{\infty}$, and hence, $e_{u} \in \mathcal{L}_{\infty}$. The closed-loop error system can then be used to conclude that the remaining signals are bounded.

Remark 2. If the system dynamics are such that $\|\tilde{N}\|$ is linear in $\|z\|$, then the function $\rho$ can be selected to be a constant, i.e., $\rho(\|z\|)=\bar{\rho}, \forall z \in \mathbb{R}^{4 n}$ for some known $\bar{\rho}>0$. In this case, the gain condition in 26 reduces to

$$
k_{s}>\frac{\bar{\rho}}{2 \sigma}
$$

and the result is global in the sense that $\mathscr{D}=\mathcal{S}_{\mathscr{D}}=\mathbb{R}^{3 n+4}$.

\section{CONCLUSION}

This paper presents a robust controller for uncertain nonlinear systems which include simultaneous time-varying state and input delays, as well as sufficiently smooth additive bounded disturbances. The controller utilizes a robust design approach to compensate for the unknown state delays coupled with an error system structure that provides a delay-free open-loop error system. The controller and LK functionals guarantee uniformly ultimately bounded tracking provided the rates of the delays are sufficiently slow. The control development can be applied when there is uncertainty in the system dynamics and when the state delay is unknown; however, the controller is based on the assumption that the time-varying input delay is known. Simulation results point to the possibility that different control or analysis methods could be developed to eliminate the assumption that the input delay is known. That is, perhaps the interval of previous control values could somehow be designed big enough to provide predictive properties despite uncertainty in the input delay.

\section{REFERENCES}

[1] M. Krstic, Delay Compensation for Nonlinear, Adaptive, and PDE Systems. Springer, 2009.

[2] J. Chiasson and J. Loiseau, Applications of time delay systems, ser Lecture notes in control and information sciences. Springer, 2007.

[3] K. Gu, V. L. Kharitonov, and J. Chen, Stability of Time-delay systems. Birkhauser, 2003.

[4] O. M. Smith, "A controller to overcome deadtime," ISA J., vol. 6, pp. 28-33, 1959.

[5] Z. Artstein, "Linear systems with delayed controls: A reduction," IEEE Trans. Autom. Control, vol. 27, no. 4, pp. 869-879, 1982.

[6] A. Manitius and A. Olbrot, "Finite spectrum assignment problem for systems with delays," IEEE Trans. Autom. Control, vol. 24, no. 4, pp. 541-552, 1979.

[7] N. Bekiaris-Liberis and M. Krstic, "Stabilization of linear strict feedback systems with delayed integrators," Automatica, vol. 46, pp. 1902-1910, 2010.

[8] M. Jankovic, "Forwarding, backstepping, and finite spectrum assignment for time delay systems," Automatica, vol. 45, pp. 2-9, 2009.

[9] _ - "Recursive predictor design for state and output feedback controllers for linear time delay systems," Automatica, vol. 46, pp. 510-517, 2010. 
[10] M. Nihtila, "Adaptive control of a continuous-time system with timevarying input delay," IEEE Trans. Autom. Control, vol. 12, pp. 357-364, 1989.

[11] R. Lozano, P. Castillo, P. Garcia, and A. Dzul, "Robust prediction-based control for unstable delay systems: Application to the yaw control of a mini-helicopter," Automatica, vol. 40, no. 4, pp. 603-612, 2004.

[12] D. Yue and Q.-L. Han, "Delayed feedback control of uncertain systems with time-varying input delay," Automatica, vol. 41, no. 2, pp. 233-240, 2005.

[13] Z. Wang, P. Goldsmith, and D. Tan, "Improvement on robust control of uncertain systems with time-varying input delays," IET Control Theory Appl., vol. 1, no. 1, pp. $189-194,2007$.

[14] J.-P. Richard, "Time-delay systems: an overview of some recent advances and open problems," Automatica, vol. 39, no. 10, pp. 1667 1694, 2003.

[15] M. Krstic, "Lyapunov stability of linear predictor feedback for timevarying input delay," IEEE Trans. Autom. Control, vol. 55, pp. 554-559, 2010.

[16] S. S. Ge, F. Hong, and T. H. Lee, "Adaptive neural control of nonlinear time-delay systems with unknown virtual control coefficients," IEEE Trans. Syst. Man Cybern. Part B Cybern., vol. 34, no. 1, pp. 499-516, 2004.

[17] S. Ge, F. Hong, and T. Lee, "Robust adaptive control of nonlinear systems with unknown time delays," Automatica, vol. 41, no. 7, pp. 1181-1190, Jul. 2005.

[18] S. J. Yoo, J. B. Park, and C. H. Choi, "Adaptive dynamic surface control for stabilization of parametric strict-feedback nonlinear systems with unknown time delays," IEEE Trans. Autom. Control, vol. 52, no. 12, pp. 2360-2365, 2007.

[19] C.-C. Hua, X.-P. Guan, and G. Feng, "Robust stabilisation for a class of time-delay systems with triangular structure," IET Control Theory Appl., vol. 1 , no. 4, pp. 875-879, 2007.

[20] H. Wu, "Adaptive robust state observers for a class of uncertain nonlinear dynamical systems with delayed state perturbations," IEEE Trans. Automat. Control, vol. 54, no. 6, pp. 1407-1412, 2009.

[21] M. Wang, B. Chen, and S. Zhang, "Adaptive neural tracking control of nonlinear time-delay systems with disturbances," Int. J. Adapt Control Signal Process., vol. 23, pp. 1031-1049, 2009.

[22] S.-C. Tong and N. Sheng, "Adaptive fuzzy observer backstepping control for a class of uncertain nonlinear systems with unknown time-delay," Int.. J. Autom. and Comput., vol. 7, no. 2, pp. 236-246, 2010.

[23] A. Kuperman and Q.-C. Zhong, "Robust control of uncertain nonlinear systems with state delays based on an uncertainty and disturbance estimator," Int. J. Robust Nonlinear Control, vol. 21, pp. 79-92, 2011.

[24] H. Huang and D. Ho, "Delay-dependent robust control of uncertain stochastic fuzzy systems with time-varying delay," IET Control Theory Appl., vol. 1, no. 4, pp. 1075-1085, 2007.

[25] B. Ren, S. S. Ge, T. H. Lee, and C. Su, "Adaptive neural control for a class of nonlinear systems with uncertain hysteresis inputs and timevarying state delays," IEEE Trans. Neural Netw., vol. 20, pp. 1148-1164, 2009.

[26] S. J. Yoo and J. B. Park, "Neural-network-based decentralized adaptive control for a class of large-scale nonlinear systems with unknown timevarying delays," IEEE Trans. Syst. Man Cybern., vol. 39, no. 5, pp. 1316-1323, 2009.

[27] M. Wang, S. S. Ge, and K. Hong, "Approximation-based adaptive tracking control of pure-feedback nonlinear systems with multiple unknown time-varying delays," IEEE Trans. Neur. Netw., vol. 21, no. 11, pp. 1804 $1816,2010$.

[28] Y. Niu, D. W. C. Ho, and J. Lam, "Robust integral sliding mode control for uncertain stochastic systems with time-varying delay," Automatica, vol. 41, pp. 873-880, 2005.

[29] W. Chen, L. Jiao, J. Li, and R. Li, "Adaptive nn backstepping outputfeedback control for stochastic nonlinear strict-feedback systems with time-varying delays," IEEE Trans. Syst. Man Cybern., vol. 40, no. 3 , pp. 939-950, 2010.

[30] B. Mirkin and P.-O. Gutman, "Robust adaptive output-feedback tracking for a class of nonlinear time-delayed plants," IEEE Trans. Automat. Control, vol. 55, no. 10, pp. 2418-2424, 2010.

[31] B. Mirkin, P.-O. Gutman, Y. Shtessel, and C. Edwards, "Continuous decentralized MRAC with sliding mode of nonlinear delayed dynamic systems," in IFAC World Congr., Milano, Italy, 2011.

[32] N. Sharma, S. Bhasin, Q. Wang, and W. E. Dixon, "RISE-based adaptive control of a control affine uncertain nonlinear system with unknown state delays," IEEE Trans. Automat. Control, vol. 57, no. 1, pp. 255-259, Jan. 2012.
[33] N. Fischer, R. Kamalapurkar, N. Sharma, and W. E. Dixon, "Rise-based control of an uncertain nonlinear system with time-varying state delays," in Proc. IEEE Conf. Decis. Control, Maui, HI, Dec. 2012, pp. 35023507.

[34] F. Mazenc and S.-I. Niculescu, "Generating positive and stable solutions through delayed state feedback," Automatica, vol. 47, pp. 525-533, 2011

[35] I. Karafyllis, "Stabilization by means of approximate predictors for systems with delayed input," SIAM J. Control Optim., vol. 49, no. 3, pp. 1100-1123, 2011.

[36] M. Krstic and A. Smyshlyaev, "Backstepping boundary control for firstorder hyperbolic PDEs and application to systems with actuator and sensor delays," Syst. Control Lett., vol. 57, no. 9, pp. 750-758, 2008.

[37] D. Bresch-Pietri and M. Krstic, "Adaptive trajectory tracking despite unknown input delay and plant parameters," Automatica, vol. 45, no. 9 , pp. 2074-2081, 2009.

[38] F. Mazenc, S. Mondie, R. Francisco, P. Conge, I. Lorraine, and F. Metz, "Global asymptotic stabilization of feedforward systems with delay in the input," IEEE Trans. Autom. Control, vol. 49, (5), pp. 844-850, 2004.

[39] F. Carravetta, P. Palumbo, and P. Pepe, "Quadratic optimal control of linear systems with time-varying input delay," in Proc. IEEE Conf. Decis. Control, 2010, pp. 4996-5000.

[40] B. Chen, X. Liu, and S. Tong, "Robust fuzzy control of nonlinear systems with input delay," Chaos, Solitons \& Fractals, vol. 37, no. 3, pp. 894-901, 2008.

[41] F. Mazenc, S. Niculescu, and M. Bekaik, "Stabilization of time-varying nonlinear systems with distributed input delay by feedback of plant's state," Automatic Control, IEEE Transactions on, vol. PP, no. 99, p. 1, 2012.

[42] P. Pepe, Z.-P. Jiang, and E. Fridman, "A new Lyapunov-Krasovskii methodology for coupled delay differential and difference equations," Int. J. Control, vol. 81, pp. 107-115, 2008.

[43] M. Krstic, "Input delay compensation for forward complete and strictfeedforward nonlinear systems," IEEE Trans. Autom. Control, vol. 55, pp. 287-303, Feb. 2010.

[44] N. Sharma, S. Bhasin, Q. Wang, and W. E. Dixon, "Predictor-based control for an uncertain Euler-Lagrange system with input delay," Automatica, vol. 47, no. 11, pp. 2332-2342, 2011.

[45] B. Castillo-Toledo, S. Di Gennaro, and G. Castro, "Stability analisys for a class of sampled nonlinear systems with time-delay," in Proc. IEEE Conf. Decis. Control, 2010, pp. $1575-1580$.

[46] S. Obuz, E. Tatlicioglu, S. C. Cekic, and D. M. Dawson, "Predictorbased robust control of uncertain nonlinear systems subject to input delay," in IFAC Workshop on Time Delay Syst., vol. 10, no. 1, 2012, pp. 231-236.

[47] X. Jiao, J. Yang, and Q. Li, "Adaptive control for a class of nonlinear systems with time-varying delays in the state and input," J. Control Theory Appl., vol. 9, pp. 183-188, 2011.

[48] J. Liu, J. Zhang, Y. Zheng, and M. He, "Robust H-infinity control for discrete-time T-S fuzzy systems with input delay," J. Control Theory Appl., vol. 9, pp. 189-194, 2011.

[49] I. Karafyllis, "Finite-time global stabilization by means of time-varying distributed delay feedback," SIAM J. Control Optim., vol. 45, pp. 320342, 2006.

[50] N. Bekiaris-Liberis and M. Krstic, "Compensation of time-varying input delay for nonlinear systems," in Mediterr. Conf. Control and Autom., Corfu, Greece, 2011.

[51] _ "Compensation of time-varying input and state delays for nonlinear systems," J. Dyn. Syst. Meas. Control, vol. 134, no. 1, p. 011009 , 2012.

[52] N. Fischer, R. Kamalapurkar, N. Fitz-Coy, and W. E. Dixon, "Lyapunovbased control of an uncertain Euler-Lagrange system with time-varying input delay," in Proc. Am. Control Conf., Montréal, Canada, June 2012, pp. 3919-3924.

[53] C. Makkar, G. Hu, W. G. Sawyer, and W. E. Dixon, "Lyapunov-based tracking control in the presence of uncertain nonlinear parameterizable friction," IEEE Trans. Autom. Control, vol. 52, pp. 1988-1994, 2007.

[54] C. Makkar, W. E. Dixon, W. G. Sawyer, and G.H, "A new continuously differentiable friction model for control systems design," in Proc. IEEE/ASME Int. Conf. Adv. Intell. Mechatron., Monterey, CA, July 2005, pp. 600-605.

[55] R. Kamalapurkar, J. A. Rosenfeld, J. Klotz, R. J. Downey, and W. E. Dixon. (2014) Supporting lemmas for RISE-based control methods. arXiv:1306.3432v3.

[56] H. K. Khalil, Nonlinear Systems, 3rd ed. Upper Saddle River, NJ, USA: Prentice Hall, 2002. 